Document downloaded from:

http://hdl.handle.net/10251/35986

This paper must be cited as:

Carbonell Olivares, J.; Torrent Martí, D.; Sánchez-Dehesa Moreno-Cid, J. (2013). Radial Photonic Crystal Shells and Their Application as Resonant and Radiating Elements. IEEE Transactions on Antennas and Propagation. 61(2):755-767. doi:10.1109/TAP.2012.2225015.

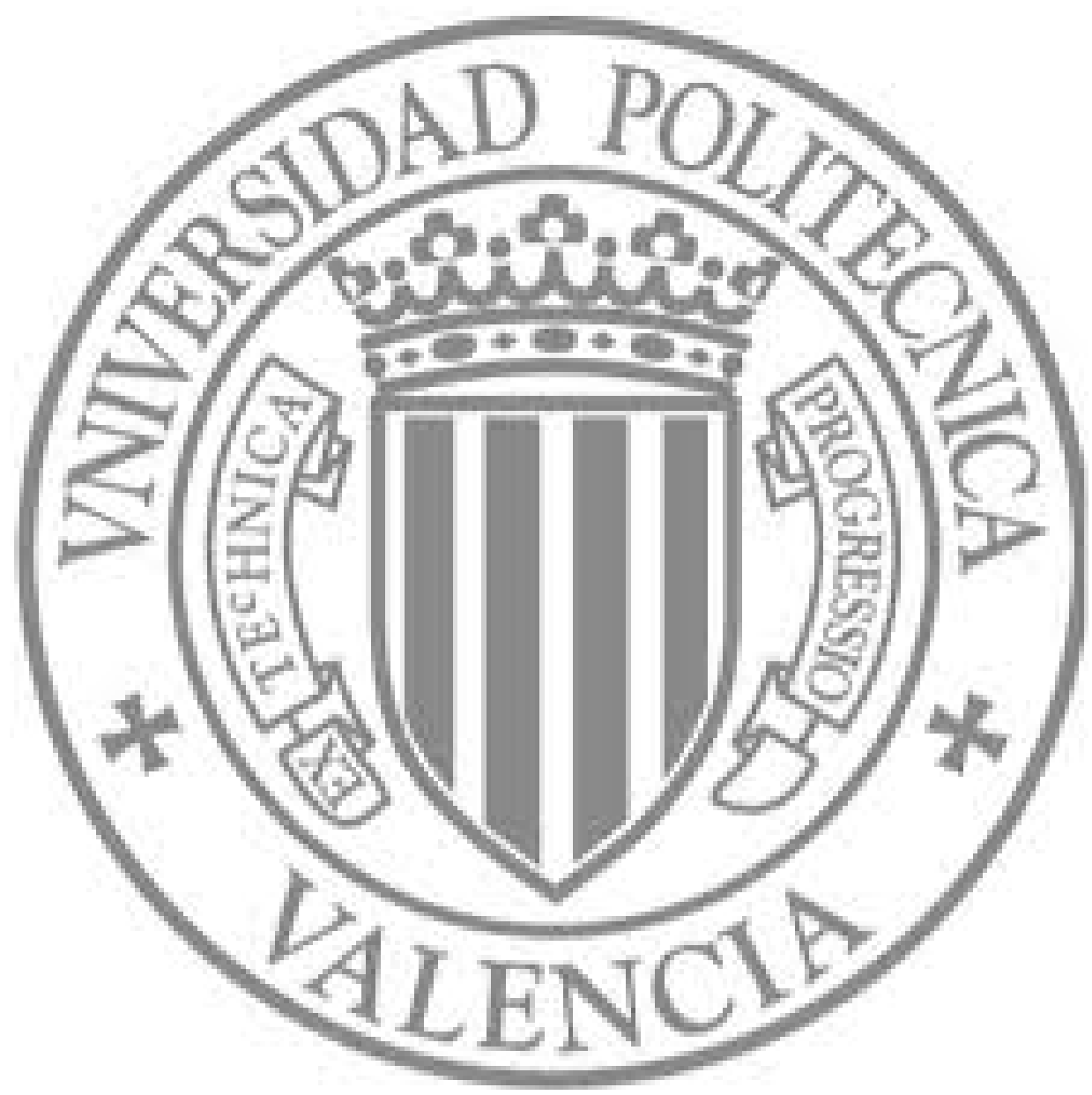

The final publication is available at

http://dx.doi.org/10.1109/TAP.2012.2225015

Copyright Institute of Electrical and Electronics Engineers (IEEE) 


\title{
Radial Photonic Crystal shells and their application as resonant and radiating elements
}

\author{
Jorge Carbonell, Daniel Torrent, and José Sánchez-Dehesa
}

\begin{abstract}
Radial Photonic Crystals (RPCs) are a class of microstructured media that possess very particular electromagnetic features. Their properties are driven by radially dependent permittivity and permeability profiles that are also anisotropic. A two dimensional RPC structure is designed and analyzed in order to assess its principal characteristics. This is done through the study of the dispersion diagram of the periodic multilayered structure and also through the analysis of a finite size device. In the later case, research is performed in view of possible applications of RPCs as resonant devices and also when they are combined with line sources. Finally, a device with reduced complexity is designed and implemented by means of a microstructured resonator array. This device is numerically analyzed and the comparison of two independent models shows very good agreement. Potential use of RPCs as frequency and location sensors is pointed out.
\end{abstract}

Index Terms-Metamaterials, scattering, point source, split ring resonators, anisotropic multilayers.

\section{INTRODUCTION}

$\mathrm{D}$ URING the last decade, a great research effort has concentrated on controlling electromagnetic wave propagation in microstructured media. These microstructured media have been named metamaterials, since they can produce unusual propagation phenomena by basically deforming the light propagation paths at will, [1-3]. This technology is based on the use of sub-wavelength inclusions that enable the interaction of electromagnetic waves to be described by effective (constitutive) material parameters. Therefore, it is possible to create materials with unusual wave propagation characteristics. These microstructured media have permitted the design of electromagnetic cloaks, negative refraction devices, omni-directional electromagnetic absorbers or lenses with focusing characteristics beating the classic $\lambda / 2$ diffraction limit, [4-7].

Microstructured devices can be designed by properly arranging different types of building units, which can be resonant or not, [8-10]. Depending on the type of arrangement employed, they can also be selected to produce isotropic or

Manuscript received April 12, 2012, revised September 25, 2012. This work was supported in part by the Spanish Ministry of Science MICINN under Grants TEC 2010-19751 and Consolider CSD2008-00066.

J. Carbonell, D. Torrent and J. Sánchez-Dehesa are with the Wave Phenomena Group (Edificio 7F), Universitat Politecnica de Valencia Camino de Vera, s/n, 46022 Valencia (Spain). (phone: +34-96-3879607; fax: +34-963877609; e-mails: jorcarol@upvnet.upv.es, datorma1@upvnet.upv.es jsdehesa@upvnet.upv.es). anisotropic behaviors [11-13], and they can even be combined with active elements for tuning or loss compensation, [14-16]. Nevertheless, frequency dispersion (i.e. limited bandwidth) and losses remain the main drawbacks still challenging this technology.

In this context, periodic, semi-periodic and/or multilayered microstructures have been studied to satisfy different types of goals, depending on the desired spatial configuration (1D, 2D or 3D). These studies are usually based on the analysis of the desired propagation characteristics at the macroscopic level, and of the elementary constituent cells at the microscopic level $[4,17,18]$. In particular, the selection of the unit cell element and arrangement is inherently linked to the targeted spatial configuration, wave polarization, operation frequency and dispersive characteristics, and isotropic or anisotropic behavior, among others. Macroscopic characteristics are usually described by effective constitutive parameters [19-21], but are also described by equivalent circuit models for design and engineering environments [22, 23]. This permits the analysis in terms of propagation characteristics (allowed or forbidden modes, propagation and attenuation constants...), that can be summarized in dispersion diagrams. Additionally, other radial structures such as dielectric photonic crystals have also been investigated, [24, 25], with the target of creating isotropic bandgaps and high quality-factor $(Q)$ resonators, [26]. Nevertheless, these radial proposals were not easily described in terms of purely periodic parameters.

In this paper, we have analyzed and designed the wave propagation characteristics of a so-called Radial Photonic Crystal (RPC). This type of metamaterial-inspired structure has the original characteristic of being invariant under translation, by implementing radially dependent constitutive parameter functions in a multilayered system. Although radial crystals were initially analyzed for acoustic waves in [27, 28], clear analogies can be established between both application fields, [29], which explain some common behaviors. For a 2D configuration, the fundamental characteristic of these microstructures derives from the periodicity of the radial profile of the anisotropic constitutive parameters. This is first analyzed in Section II with the proper design equations, together with the dispersion diagram information and related propagation parameters. In Section III, moving to the more practical aspects and application potentiality, finite size structures based on these concepts are studied as resonating elements. Next, in Section IV, the excitation of RPC structures with line sources is assessed and some potential applications 
are pointed out. Finally, Section V reports a practical design of a sample structure taking into account some simplifications allowing the feasibility of the device. These limitations are imposed on the number of constitutive parameters and layers implemented. The paper ends with a conclusions section summarizing the main findings of this work.

\section{RADIAL WAVE CRYSTALS FOR ELECTROMAGNETIC WAVES}

\section{A. Design equations}

Focusing on a $2 \mathrm{D}$ configuration, a RPC can be designed in such a way as to satisfy the fundamental condition of being invariant under translation in cylindrical coordinates. We apply this case to $\mathrm{TM}^{\mathrm{z}}$ modes ( $\mathrm{E}^{\mathrm{z}}$ modes) for the $2 \mathrm{D}$ configuration. We also may assume that the electric field at an arbitrary point of the 2D space $(r, \theta)$ takes the form $E(r, \theta)=\Sigma_{q}$ $E_{q}(r) \cdot e^{i q \theta}$. Starting from the scalar Helmholtz wave propagation equation, for the radial part, we have:

$$
\left[-\frac{1}{r \varepsilon r} \frac{\partial}{\partial r}\left(\frac{r}{\mu r} \frac{\partial}{\partial r}\right)+\frac{q^{2}}{r^{2} \varepsilon r \mu r}\right] E_{q} r=\omega^{2} E_{q} r
$$

where $\varepsilon(r)$ and $\mu(r)$ are radially dependent constitutive parameter isotropic functions (see for example [30] for the derivation of this equation). Now, we are interested in obtaining a solution of (1) that is invariant under a translation of type $r \rightarrow r+n d$, with $n$ being an integer and $d$ being a periodicity parameter. To achieve this goal, the parameters $r \varepsilon(r), r / \mu(r)$ and $r \mu(r)$ need to be simultaneously periodic. However, this condition is not possible to accomplish with the couple $r / \mu(r)$ and $r \mu(r)$, unless $\mu(r)$ is a tensor with anisotropic properties. Therefore, if $\mu(r)$ has a tensorial definition with $\mu_{r}(r)$ and $\mu_{\theta}(r)$ as diagonal components, equation (1) can be re-written as (see the Appendix for details on this equation):

$$
\left[-\frac{1}{r \varepsilon_{z} r} \frac{\partial}{\partial r}\left(\frac{r}{\mu_{\theta} r} \frac{\partial}{\partial r}\right)+\frac{q^{2}}{r^{2} \varepsilon_{z} r \mu_{r} r}\right] E_{q} r=\omega^{2} E_{q} r
$$

In equation (2) terms $r / \mu_{\theta}(r)$ and $r \mu_{r}(r)$ can be made simultaneously periodic, together with $r \varepsilon_{z}(r)$, and an invariance under translation condition, as stated above, is possible.. Therefore, it is possible to apply Bloch's theorem to obtain a band structure for the radial equation. Related types of metamaterial-based studies can be found in [31] (multilayered cylinders for cloaking) and in [32] (radially inhomogeneous and anisotropic effective permittivity and permeability for cylindrical transmission-line metamaterials). Nevertheless, in these two cases the previous condition of invariance under translation is not satisfied.

An implementation of a RPC can be made by using two alternating metamaterial layers of types $a$ and $b$ with constant thicknesses $d_{a}$ and $d_{b}$ along the radial direction $\left(d=d_{a}+d_{b}\right)$. We can introduce a notation with a vector containing the constituent parameter functions $\mathbf{X}(r) \equiv\left(\mu_{r}(r)^{-1}, \mu_{d}(r), \varepsilon_{z}(r)^{-1}\right)$, that, depending on the layer type $(a$ or $b)$ and the radial

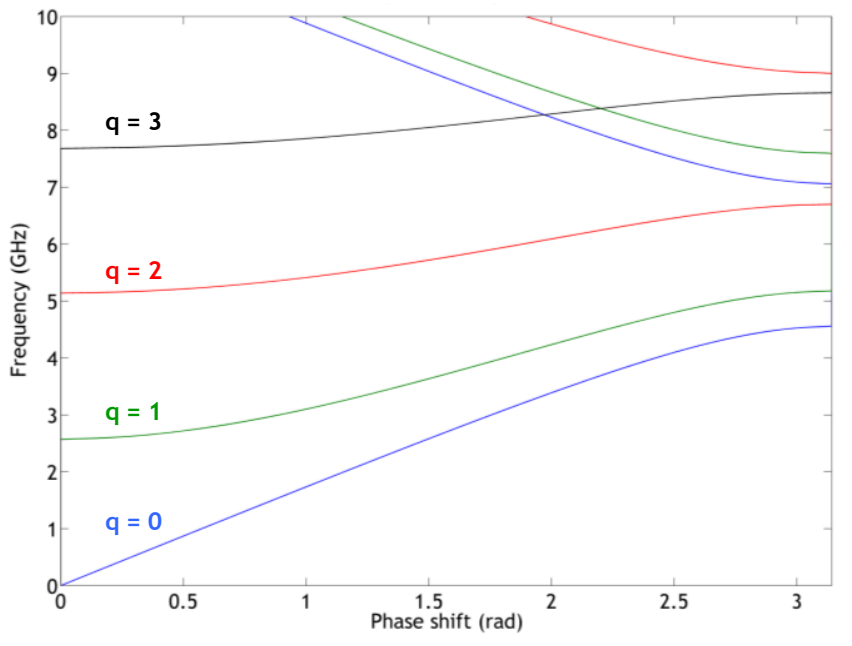

Fig. 1. Dispersion diagram for the first 4 modes of a Radial Photonic Crystal. The RPC is made from a multilayered structure of alternate layers of types $a$ and $b$ with constitutive parameters $\left(\mu_{r a}^{-1}, \mu_{\theta a}, \varepsilon_{z a}^{-1}\right)=(3.125 r / d, 0.8 r / d$ $0.12 r / d)$ and $\left(\mu_{r b}{ }^{-1}, \mu_{b b}, \varepsilon_{z}{ }^{-1}\right)=(4.175 r / d, 0.4 r / d, 0.06 r / d)$ and periodicity parameter $d=d_{a}+d_{b}=5+5 \mathrm{~mm}$.

coordinate $r$, can be expressed as:

$\mathrm{X} r=\left\{\begin{array}{l}r \hat{\mathrm{X}}_{a} \text { if } \quad n-1 d<r<n-1 d+d_{a} \\ r \hat{\mathrm{X}}_{b} \text { if } \quad n-1 d+d_{a}<r<n d\end{array}\right.$

where $n$ is an integer that takes values $n=1,2, \ldots, \infty$ and $\hat{\mathrm{X}}_{i}$ (for $i=a, b$ ) are (normalized) vectors whose components are real numbers giving the slope of the linearly dependent electromagnetic parameters, i.e. $\hat{\mathrm{X}}_{i}=\hat{\mu}_{i r}{ }^{-1}, \hat{\mu}_{i \theta}, \hat{\varepsilon}_{i z}{ }^{-1}$. By introducing $\hat{\mathrm{X}} r$ in (2), we obtain the 2D wave equations for the respective layers ( $a$ and $b$ ):

$\frac{\partial^{2} E_{q} r}{\partial r^{2}}+\left[\omega^{2} \hat{\varepsilon}_{i z} \hat{\mu}_{i \theta}-q^{2} \frac{\hat{\mu}_{i \theta}}{\hat{\mu}_{i r}}\right] E_{q} r=0$.

Both equations for each one of the layers $a$ and $b$ have plane-wave solutions with a dispersion relation given by:

$k_{i q}^{2}=\omega^{2} \hat{\varepsilon}_{i z} \hat{\mu}_{i \theta}-q^{2} \frac{\hat{\mu}_{i \theta}}{\hat{\mu}_{i r}} \quad i=a, b$.

Now, the dispersion relation for the multilayered structure can be obtained from (5) using a method similar to the one already employed in [33]. The composite relation is given by:

$$
\begin{aligned}
\cos K d= & \cos k_{a q} d_{a} \cos k_{b q} d_{b} \\
& -\frac{1}{2}\left[\frac{\hat{\mu}_{a \theta} k_{b q}}{\hat{\mu}_{b \theta} k_{a q}}+\frac{\hat{\mu}_{b \theta} k_{a q}}{\hat{\mu}_{a \theta} k_{b q}}\right] \sin k_{a q} d_{a} \sin k_{b q} d_{b}
\end{aligned}
$$

where $K$ is the Bloch wave number. 

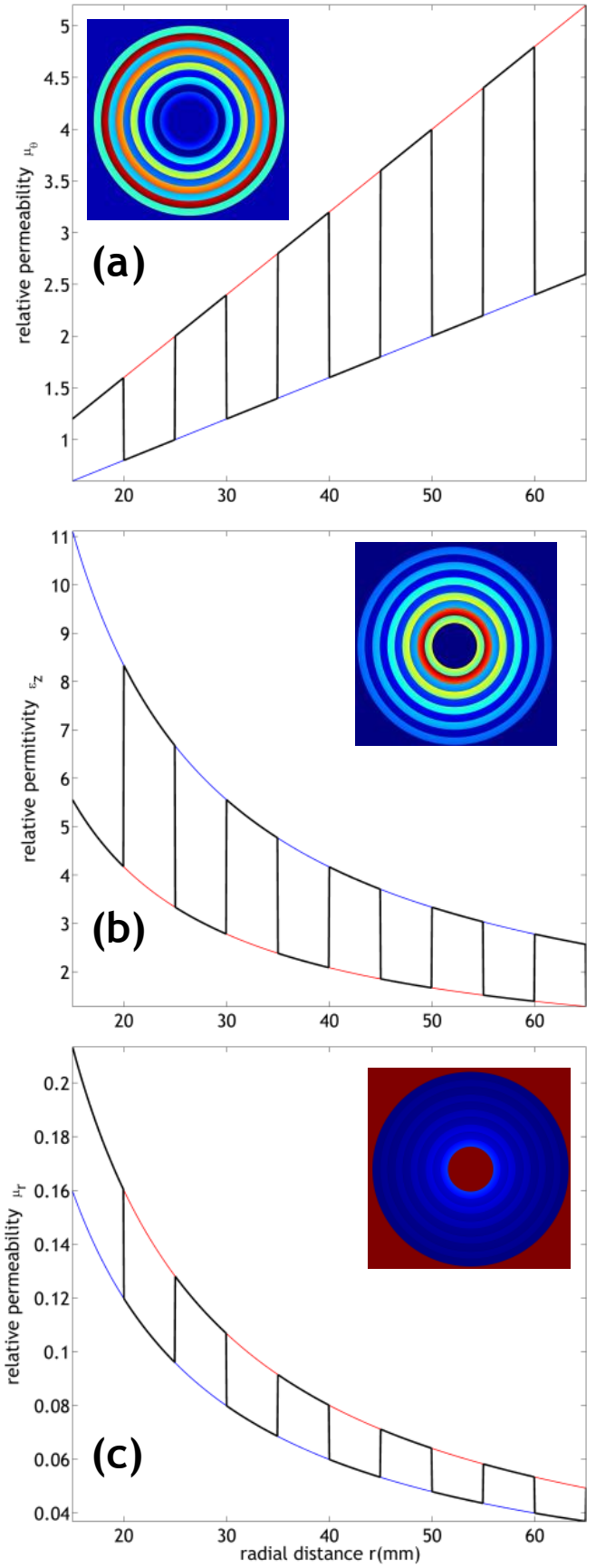

Fig. 2. Constitutive parameter profiles for a 2D Radial Photonic Crystal with stair like shapes as a function of the radial coordinate $r$ in the shell region. The origin of $r$ is located at the center of the RPC. (a) angular permeability $\mu_{\dot{\theta}}$; (b) permittivity $\varepsilon_{z}$; (c) radial permeability $\mu_{r}$. Actual parameter values (black curve) correspond to data in Fig. 1, and are bounded by the functions describing the parameter in the alternative layers of types $a$ (red curve) and $b$ (blue curve). Insets are color plots of the curve values in the device plane (high values correspond to red color and low values correspond to blue color).

Figure 1 displays the radial dispersion diagram for a RPC with constitutive parameters $\left(\mu_{r a}^{-1}, \mu_{\theta a}, \varepsilon_{z a}^{-1}\right)=(3.125 r / d$, $0.8 r / d, 0.12 r / d)$ and $\left(\mu_{r b}{ }^{-1}, \mu_{\theta b}, \varepsilon_{z b}{ }^{-1}\right)=(4.175 r / d, 0.4 r / d$, $0.06 r / d)$. The first 4 modes are included $(q=0,1,2,3)$ for a microstructure with $d=d_{a}+d_{b}=5+5 \mathrm{~mm}$. The layer

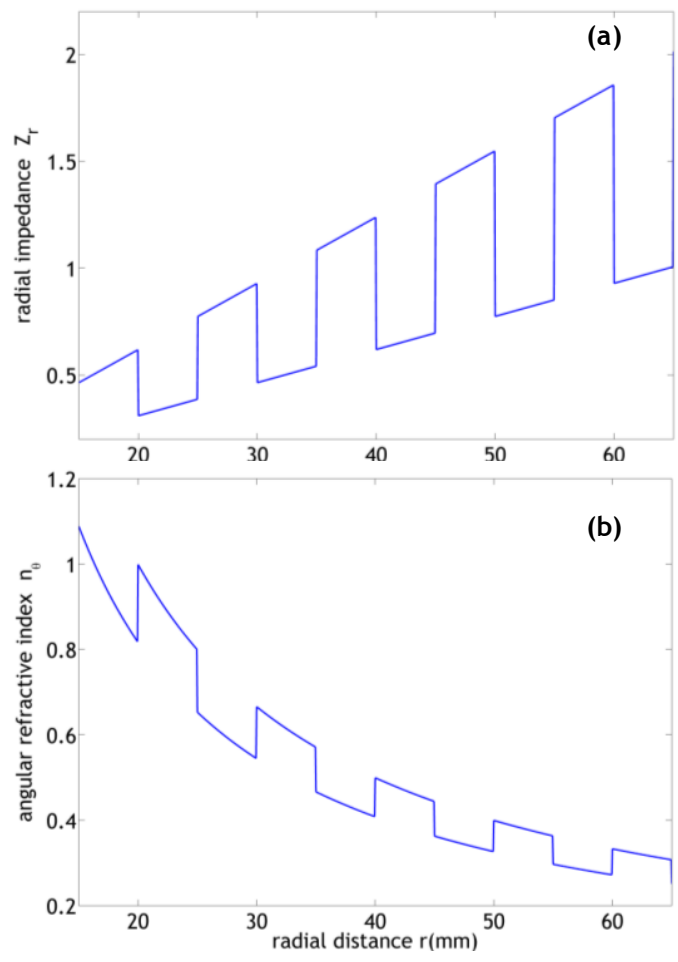

Fig. 3. Radial Photonic Crystal impedance and refractive index as a function of the radial position. (a) radial impedance $Z_{r}$; (b) angular refractive index $n_{\theta}$.

dimensions directly fix the operation frequencies, so the structure is theoretically scalable in a broad region of frequencies. Modes with $q>0$ have a low frequency band gap with no transmission, and only the $q=0$ mode has no cutoff frequency. It is interesting to note that this particular parameter selection makes the mode $q=2$ have its first branch completely included within the first band gap of mode $q=0$, and there is no other mode propagating in that frequency range. The lower frequency of the first band gap of mode $q=$ 1 matches the cutoff frequency of mode $q=2$.

\section{B. Effective constitutive parameters}

Designing a practical structure based on the concept of RPC obviously requires that the microstructure has a finite size. Let us note that the previous dispersion diagram analysis corresponds only to the periodic unit cell (infinite structure). Besides this, $\mu_{r}(r)$ and $\varepsilon_{2}(r)$ reach unbounded values close to the origin $r=0$, and $\mu_{d}(r)$ will follow a linear increase with $r$. Because of these two considerations, a practical implementation of a RPC requires a finite number of concentric layers and also a void (inner) cavity in its center. By taking the vector parameters $\mathbf{X}(r)$ defined previously and an inner cavity radius of $r_{i n t}=15 \mathrm{~mm}$, we have generated the constitutive parameters corresponding to a device with ten layers (five layers of type $a$ and five layers of type $b$ ). They are displayed in Fig. 2. Curves for $\mu_{r}(r), \mu_{d}(r)$, and $\varepsilon_{z}(r)$ are displayed as a function of the radial distance from the center of the microstructure and for the area occupied by the RPC shell (15 to $65 \mathrm{~mm}$ radius). Let us note that the permittivity and permeability functions have stair-like shapes due to the 
TABLE I

RESONANT FREQUENCIES FOR A RPC WITH TEN LAYERS

\begin{tabular}{|c|c|c|}
\hline $\begin{array}{l}\text { Mode } \\
\text { pattern }\end{array}$ & $\begin{array}{c}\text { Mode } \\
\text { symmetry }\end{array}$ & $\begin{array}{c}\text { Resonant } \\
\text { frequency }(\mathrm{GHz})\end{array}$ \\
\hline & $\begin{array}{l}\text { shell Bragg } \\
q=1 m=1\end{array}$ & 2.712 \\
\hline & $\begin{array}{l}\text { shell Bragg } \\
q=0 m=3\end{array}$ & 2.800 \\
\hline & $\begin{array}{l}\text { shell Bragg } \\
q=1 m=2\end{array}$ & 3.160 \\
\hline & $\begin{array}{l}\text { shell Bragg } \\
q=1 m=3\end{array}$ & 3.880 \\
\hline & $\begin{array}{l}\text { shell Bragg } \\
q=0 m=4\end{array}$ & 3.895 \\
\hline & $\begin{array}{l}\text { shell Bragg } \\
q=1 \mathrm{~m}=4\end{array}$ & 4.706 \\
\hline & $\begin{array}{c}\text { inner cavity } \\
q=0\end{array}$ & 4.942 \\
\hline & $\begin{array}{l}\text { shell Bragg } \\
q=2 m=1\end{array}$ & 5.226 \\
\hline & $\begin{array}{l}\text { shell Bragg } \\
q=2 m=2\end{array}$ & 5.487 \\
\hline & $\begin{array}{l}\text { shell Bragg } \\
q=2 m=3\end{array}$ & 5.911 \\
\hline 8 & $\begin{array}{c}\text { inner cavity } \\
\quad q=1\end{array}$ & 6.343 \\
\hline
\end{tabular}

Resonant modes cover only the frequency range from 2.7 to $6.5 \mathrm{GHz}$.

fact that each parameter is alternating between layers $a$ and $b$ that follow the two linearly increasing (or inversely decreasing) functions of each layer type. Insets show 2D schemes of how the values of each parameter are distributed on the RPC shell surface. Background values inside the inner (void) cavity and outside the shell border are $\varepsilon=\mu=1$. The values selected for the three parameters remain in any case within ranges that can be achieved with metamaterial-inspired units based for example on micro-resonators and are not negative.

From the definition of the three constitutive parameters, two propagation parameters can be derived: impedance and refractive index. In the cylindrical coordinate system both $Z$ and $n$ can be defined for the two possible propagation directions, with radial and angular components. The reduced impedance $Z$ can be calculated as:

$Z_{r}=\sqrt{\frac{\mu_{\theta}}{\varepsilon_{z}}}=r \sqrt{\frac{\hat{\mu}_{\theta}}{\hat{\varepsilon}_{z}}}$

$Z_{\theta}=\sqrt{\frac{\mu_{r}}{\varepsilon_{z}}}=\sqrt{\frac{\hat{\mu}_{r}}{\hat{\varepsilon}_{z}}}$.

Also, refractive index $n$ is directly obtained from the constitutive parameters:

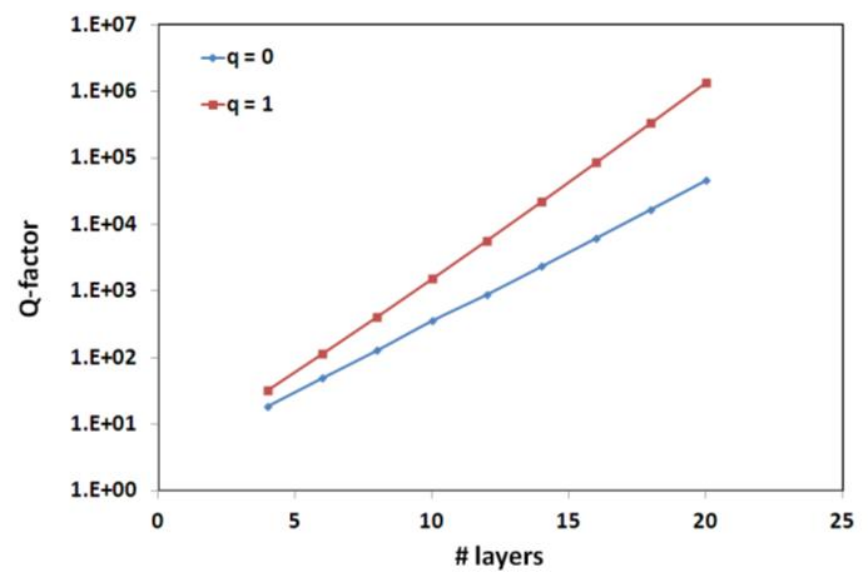

Fig. 4. $Q$-factors for the inner cavity resonances with symmetries $q=0\left(f_{0}=\right.$ $4.94 \mathrm{GHz})$ and $q=1\left(f_{1}=6.34 \mathrm{GHz}\right)$, as a function of the number of concentric layers forming the RPC shell. Below 4 layers, resonant modes disappear.

$$
\begin{aligned}
& n_{\theta}=\sqrt{\varepsilon_{z} \mu_{r}}=\frac{1}{r} \sqrt{\hat{\mu}_{r} \hat{\varepsilon}_{z}} \\
& n_{r}=\sqrt{\varepsilon_{z} \mu_{\theta}}=\sqrt{\hat{\mu}_{\theta} \hat{\varepsilon}_{z}} .
\end{aligned}
$$

The definition of the constitutive parameters determines that $Z_{\theta}$ alternates between two constant values in each layer type (equation (7)) and $n_{r}$ is constant over the whole shell surface (equation (8)). $Z_{\theta}$ and $n_{r}$ are not radially dependent. $Z_{r}$ and $n_{\theta}$ are displayed in Fig. 3, where it is possible to observe that both parameters have a radial dependence. The dependence is linearly increasing for the impedance and inversely decreasing for the refractive index. Both parameters $Z$ and $n$ are positive and they have values close to unity, ensuring matching with the background.

\section{RESONATOR STRUCTURES}

A resonator is analyzed as the first application of a finite size RPC structure. RPC structures were studied as acoustic resonators in [28], by evaluating the resonance field patterns and their characteristics. Here, we have used a commercial software package based on finite elements, [34], to compute the resonant frequencies (eigen-frequencies) of the 10 layer RPC defined in Fig. 2.

Table I displays the resonant mode patterns, mode symmetries and resonant frequencies of all resonant modes in the frequency range from $2.7 \mathrm{GHz}$ to $6.5 \mathrm{GHz}$. On the one hand, exact resonance frequencies are determined by the RPC shell. On the other hand, frequency ranges where it is possible to observe each symmetry type are driven by the dispersion diagram of the infinite structure (see Fig. 1). Comparing the resonant frequencies of each mode and symmetry pattern with the corresponding branches in the dispersion diagram of Fig. 1 , it is possible to confirm the co-existence of modes $q=0$ and $q=1$ between roughly $2.5 \mathrm{GHz}$ and $4.5 \mathrm{GHz}$. Also, above 5.2 $\mathrm{GHz}$ (and up to the upper considered frequency $6.5 \mathrm{GHz}$ ), the only allowed mode in the resonant RPC shell has a $q=2$ 
TABLE II

QUALITY FACTORS FOR DIFFERENT SHELL TYPES OF EQUAL SIZE

\begin{tabular}{lcc}
\hline \multicolumn{1}{c}{ Type of shell } & Q-factor & $\begin{array}{c}\text { Resonant } \\
\text { frequency }(\mathrm{GHz})\end{array}$ \\
\hline I. full RPC & 335 & $4.94(q=0)$ \\
& 1517 & $6.34(q=1)$ \\
II. only $\varepsilon_{z}(r)$ profile & 12 & $5.87(q=0)$ \\
$\left(\mu_{\theta}=\mu_{r}=1\right)$ & 100 & $8.43(q=1)$ \\
III. mean $\varepsilon_{z}=3.56$ & 12 & $5.99(q=0)$ \\
$\left(\mu_{\theta}=\mu_{r}=1\right)$ & 18 & $9.46(q=1)$ \\
IV. Bragg fiber & & $5.24(q=0)$ \\
$\left(n_{a}=3 ; n_{b}=1.5 ; n_{c}=1\right)$ & 78 &
\end{tabular}

Resonant modes $q=0$ and $q=1$ for an inner cavity of size $r_{i n t}=15 \mathrm{~mm}$ and an external shell radius $r_{\text {ext }}=65 \mathrm{~mm}$. This is equivalent to a ten layers RPC shell in size. Type IV Bragg fiber has same inner cavity size (hollow core) and same number of layers (10), although external radius is smaller.

symmetry. These shell resonances are based on Bragg effects caused by the finite size of the structure in terms of radial and angular dimensions.

Together with these shell resonances, the inner cavity resonances are also represented. The position of these inner cavity resonances is driven by the inner cavity radius and also by the composition (i.e. constituent parameter functions) of the RPC shell. Fig. 4 displays, for the inner cavity resonances, the evaluated quality factor variation as a function of the number of layers of the device. The only source of loss considered for this calculation is radiation loss associated to the "open" boundary surrounding the whole simulation domain (i.e. radiation loss). From the imaginary part of the eigenfrequency it is possible to obtain the quality factor of the resonant mode. The calculated quality factor $Q$ is related to radiation $\left(Q_{r a d}\right)$ and not to dissipation $\left(Q_{d i s}\right)$. The results show that quality factors for the inner cavity resonances $(q=0$ at $4.94 \mathrm{GHz}$ and $q=1$ at $6.34 \mathrm{GHz}$ ) increase exponentially as the multilayered RPC radius increases. This is equivalent to increasing the size of the RPC shell in steps of one pair of layers. Resonances with higher frequencies have higher $Q$ factors. If the size of the RPC shell goes below 4 layers, then the inner cavity resonance disappears, since the shell is not able to confine the resonant mode any more. This result is equivalent to that of the acoustic counterpart of the RPC, [28]. It is also similar to the behavior observed in resonances associated to localized effects in photonic crystals, [35]. As isolation between the inner cavity and the external background increases, a higher $Q$-factor is obtained.

This study would be incomplete if a RPC microstructure is not compared to other types of shells with equivalent size. Table II displays the $Q$-factors obtained for the two resonant modes of the inner cavity for four cases of shells. All four have identical size with an inner radius (equal to the inner cavity radius) $r_{i n t}=15 \mathrm{~mm}$ and an outer radius $r_{\text {ext }}=65 \mathrm{~mm}$. This exactly corresponds to the RPC dimensions given in Fig. 2 (ten layers device). Type I is a full RPC (with three constitutive parameter profiles as in Fig. 2), which shows the highest $Q$-factors for both resonant frequencies as compared to the other. The second shell configuration (Type II) has identical $\varepsilon_{z}(r)$ profile and constant permeability parameters $\mu_{\theta}$ $=\mu_{r}=1$. The third case (Type III) is a simple dielectric shell of permittivity equal to the mean permittivity value of the previous two designs $\left(\varepsilon_{z}=3.56\right)$. The $Q$-factors decrease for Types II and III and at the same time the resonant frequencies for the inner cavity modes increase, although the inner cavity size does not vary between configurations. The $Q$-factor values obtained for the full RPC are comparable with the ones obtained for the Circular Photonic Crystals of [26], taking into account that these devices were designed with alumina rods of high permittivity $\left(\varepsilon_{r}=9.1\right)$. Finally, Type IV is a Bragg multilayered fiber with a hollow core (refractive index $n_{c}=1$ ) of same size as the inner cavity of the RPC. This Bragg fiber has a multilayered cladding with alternating type-a and type-b layers. Their refractive indexes are set respectively, for comparison purposes, to $n_{a}=3$ and $n_{b}=1.5$. The thickness of the layers in the cladding section satisfies the quarter-wave stack condition, as analyzed in $[36,37]$.

\section{COMBINATION WITH LINE SOURCES}

Besides their use as resonant structures, we have studied the interaction of line sources when they are combined with RPC microstructures and they illuminate them. Since we are studying $2 \mathrm{D}$ configurations, a point source in the $2 \mathrm{D}$ plane is assimilated to a vertically oriented line source, i.e. parallel to the $z$-axis in the $(r, \theta, z)$ coordinate system. A normalized source of $1 \mathrm{~A}$ current is used for all cases in what follows. The simulation domains are bounded with radiation boundaries, mimicking an open problem.

Numerical results obtained with the full-wave solver have been obtained in several configurations, where the combination of the source and the RPC exhibits substantially different conceptual behaviors. In our case, we have basically evaluated the interaction between both elements in two situations. First, we have analyzed the interaction with the line source when it is located inside the inner cavity, and illuminates the device at a resonance frequency of the RPC shell (Bragg resonance). Second, we have illuminated the RPC externally with the line source located at a distance larger than $r_{\text {ext }}$ from the center of the structure. An example of each one of these configurations is displayed in Fig. 5. These are plots of the E-field magnitude with a line source illuminating a ten layer RPC microstructure with $r_{i n t}=15 \mathrm{~mm}$ and $r_{\text {ext }}=65 \mathrm{~mm}$. In Fig. 5(a), the line source is located at a position $x_{a}=y_{a}=6$ $\mathrm{mm}$, i.e. within the inner cavity of the RPC (origin of coordinates is at the RPC center). In Fig. 5(b), the line source is located at a position $x_{b}=y_{b}=75 \mathrm{~mm}$. In both plots, the line sources excite the RPCs at a frequency $f=5.91 \mathrm{GHz}$, which corresponds to a $q=2$ order resonant mode (see Table I).

Figure 5(a), clearly demonstrates that if the source frequency corresponds to a Bragg shell resonance, the shell strongly modifies the radiation pattern of the line source. Instead of having an omni-directional radiation pattern, it is changed to a quadrupolar pattern matching the mode symmetry of the device. This result demonstrates that RPC 


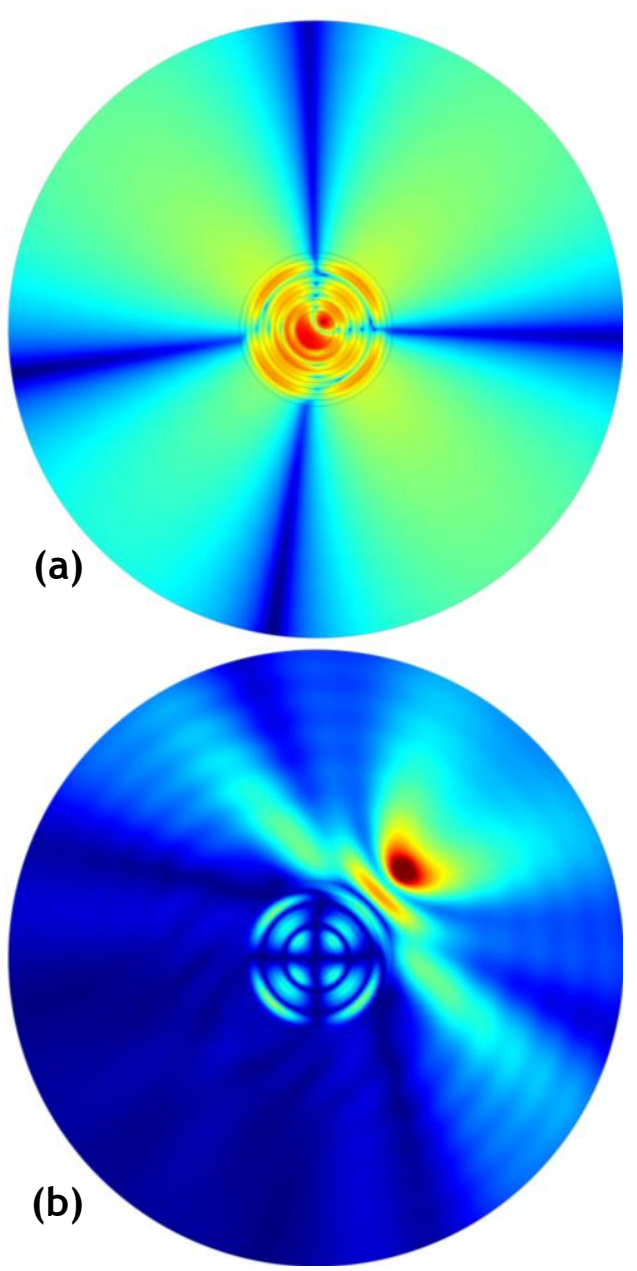

Fig. 5. E-field magnitude plot for a ten layers RPC device illuminated by a line source; (a) line source located at position $x_{a}=y_{a}=6 \mathrm{~mm}$ from the RPC center, i.e. inside the central cavity; (b) line source located at position $x_{b}=y_{b}$ $=75 \mathrm{~mm}$ from the RPC center, i.e. external illumination. In both cases the line source emits at $f=5.91 \mathrm{GHz}$, corresponding to a $q=2$ symmetry resonant mode.

shells can be a powerful tool to tailor the radiation pattern of omni-directional sources with different shapes according to the resonant mode distribution. The radiation patterns will have a frequency dependent behavior, closely driven by the symmetry of the shell resonance patterns. As a general consideration, at frequencies around each shell resonance frequency the radiation pattern of the line source will still be transformed by the symmetry of the resonant shell mode. For this design, RPC shell resonances are not very sharp and have low quality factors (as opposed to the inner cavity resonances of Table II). Hence, the resonance pattern is observable in a large frequency band around the nominal resonance frequency. Roughly, the bandwidth at which the resonance pattern is significant around the nominal value is inversely related to the quality factor of each resonance. Additional simulations show that the symmetry of the $q>0$ modes precludes exciting them with a line source if the source is located exactly at the center of the inner cavity, [38]. For this reason, the line source is displaced with respect to the center in Fig. 5(a). Note also that the displaced position of the source
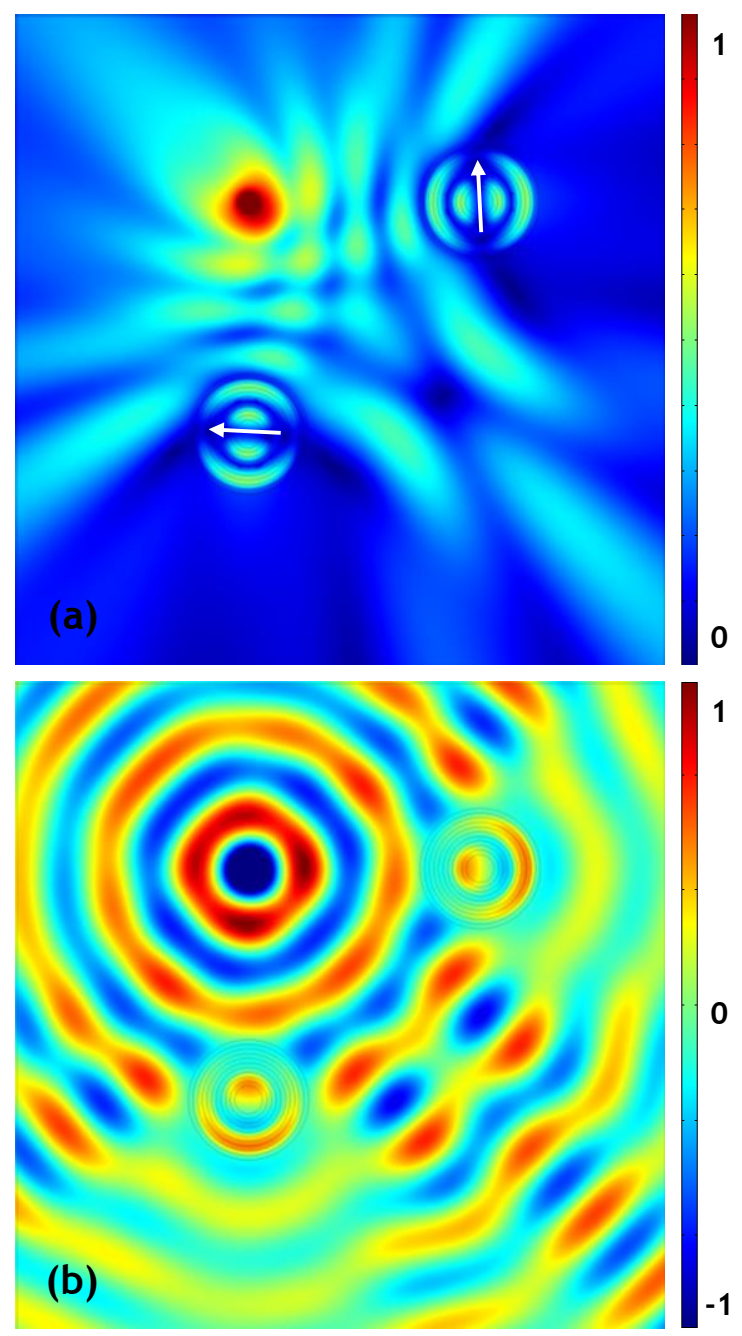

Fig. 6. Field plots in (a) magnitude of the E-field, with superimposed white arrows of dipole vectors, in (b) real part of the $\mathrm{E}^{\mathrm{z}}$ component (color scales show normalized values). Two identical RPC shells are illuminated by an external point source located at $200 \mathrm{~mm}$ of each RPC (distance to center of the devices). At a source frequency $f=3.16 \mathrm{GHz}$, a $q=1$ resonance is excited in both shells. Note the shell patterns oriented and pointing towards the source location.

with respect to the inner cavity center defines the maximum radiation directions of the combination of shell plus source. Further studies can be made on how the position of the line source in the central cavity is determinant to optimizing the radiation properties of the combined source and shell. It is anticipated that because the RPC is a "very ordered" system, radiated power of the combined system could be increased with respect to the isolated line source, as in other metamaterial combinations with radiating elements, $[32,38$, 39]. This type of analysis is out of the scope of this paper.

Figure 5(b) displays an interaction example of an external line source in close proximity to the RPC shell. It is again the same source frequency $f=5.91 \mathrm{GHz}$. Within the RPC shell the E-field pattern associated to the $q=2$ mode is clearly excited. It is therefore possible to transfer energy from the source to the RPC, taking advantage of the fact that it can trap it at the frequencies of the shell Bragg modes. At the same time, the resonance pattern excited in the shell is a proof of what 
frequency is being emitted by the external source. An additional interesting point with respect to this configuration is the fact that the symmetric mode pattern of the E-field in the device is pointing or is oriented towards the source. This effect opens up the possibility of assessing not only the frequency at which the source is emitting, but also its position.

An example to illustrate the potential application of RPCs as position sensors is given in Fig. 6. The E-field pattern of a line source illuminating a pair of identical RPC shells is depicted. Panel (a) represents the magnitude of the E-field, while panel (b) represents the real part of $E^{z}$. The source is located at a distance of $200 \mathrm{~mm}$ from each RPC center and radiates at a frequency $f=3.16 \mathrm{GHz}$. This frequency corresponds to the resonance frequency of a $q=1$ symmetry mode (from Table I). Note in this case that the symmetric mode patterns of each RPC point towards the location of the illuminating source. Especially if the symmetry of the resonant mode is $q=1$, only two RPCs could locate the exact position of a line source according to the orientation of the E-field maxima in the respective shells. If the RPCs are close one to the other, a second order interaction between them can happen: a reflected wave from one RPC excites the second RPC. This may limit the accuracy of RPCs in position sensing applications.

\section{IMPLEMENTATION USING A SET OF REDUCED PARAMETERS}

Implementing a structure with the characteristics presented in the previous sections is a difficult task. Let us recall that the full configuration of the RPC is defined by all three radially dependent constitutive parameters as displayed in Fig. $2\left(\mu_{r}(r)\right.$, $\mu_{\theta}(r)$ and $\left.\varepsilon_{z}(r)\right)$. It is an anisotropic and multilayered structure defined by two types of layers, $a$ and $b$. In order to possibly fabricate such a device we have analyzed a reduced model that should be easily fabricated.

In practice we have tried to satisfy the following generic requirements:

1) The structure should be able to act as a resonator at specific shell frequencies, as the RPC does.

2) Interaction capabilities with radiating sources should be preserved.

3) It should be feasible with standard microwave fabrication techniques.

4) Multiple layers with different constitutive parameter values should be implemented by means of discrete resonant elements.

5) Layer size and resonant element dimensions should remain within a reasonable homogenization limit depending on the operation frequency.

The target is to maintain in a large part the functionality of the ideal RPC shell but to reduce the fabrication complexity to an affordable level. Therefore instead of implementing all three constitutive parameter functions, we have only implemented a reduced parameter set. Strictly speaking, the structure will not satisfy the design equations as proposed in Section II, but it will retain in a great part the behavior of the
RPC shell with a set of reduced parameters.

\section{A. Equivalence with an ideal profile RPC}

It is possible to find an approximate equivalence between two devices with the same dimensions but with different parameter profiles. In particular, we have studied a simplified shell, where only a $\mu_{d} r$ ) profile is implemented, that can be compared with an ideal profile RPC, where all three constitutive parameters are implemented. This equivalence can be supported if we analyze again equation (2), which can be re-written as a Sturm-Liouville equation for the radial dependence of the electric field (see [40]):

$\left[-\frac{\partial}{\partial r}\left(\frac{r}{\mu_{\theta} r} \frac{\partial}{\partial r}\right)+\frac{q^{2}}{r \mu_{r} r}\right] E_{q} \quad r=r \varepsilon_{z} \quad r \quad \omega^{2} E_{q} r$.

In this equation, the eigenvalues of the problem $\omega^{2}$ are the squared resonant frequencies of the RPC shell, and functions $r \mu_{\theta}(r), 1 /\left(r \mu_{r}(r)\right)$ and $r \varepsilon_{z}(r)$ define the normalized constitutive parameters as in (3). We intend to implement a simplified shell having only a radially dependent function for the angular permeability and keeping constant values of radial permeability $\left(\mu_{r}(r)=\mu_{r_{r} \text { red }}=1\right)$ and permittivity $\left(\varepsilon_{z}(r)=\varepsilon_{z_{-} \text {red }}\right.$ $\equiv$ constant). By inspection of (9), this reduced profile shell would be equivalent to an ideal profile RPC having particular values for the radial permeability and the permittivity functions. A first order approximation of the ideal profile shell can be developed neglecting high order terms and giving the following approximate relationships:

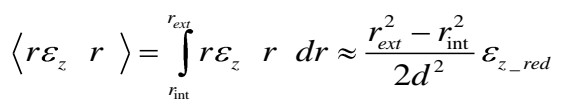

$$
\begin{aligned}
& \left\langle\frac{1}{r \mu_{r} r}\right\rangle=\int_{r_{\mathrm{int}}}^{r_{\mathrm{ext}}} \frac{d r}{r \mu_{r} r} \approx \ln \left(\frac{r_{\text {ext }}}{r_{\text {int }}}\right) \mu_{r_{-} \text {red }} .
\end{aligned}
$$

Therefore, (10) and (11) help to define an equivalence between two shells (ideal profile and reduced profile) that should have similar resonant frequencies. In the case of a four layers shell, with a periodicity parameter $d$, the normalized components of vectors $\hat{\mathrm{X}}_{i}$ (for each layer of type $i=a, b$ ) corresponding to the previous equations have to satisfy the following relationships:

$$
\begin{aligned}
& \hat{\varepsilon}_{a} \approx \frac{1}{\frac{r_{e x t}^{2}-r_{\text {itt }}^{2}}{2 d^{2}} \varepsilon_{z_{-} r e d}-\frac{1}{\hat{\varepsilon}_{b}}} \\
& \hat{\mu}_{r_{-} a} \approx \ln \left(\frac{r_{e x t}}{r_{\text {int }}}\right) \mu_{r_{-} r e d}-\hat{\mu}_{r_{-} b} .
\end{aligned}
$$

For the ideal profile shell, these equations relate the normalized components between the alternate layers and also with the reduced profile shell parameters. The third constitutive parameter $\mu_{d}(r)$ is identical in both shells. This approach allows assessing the approximate behavior of the 


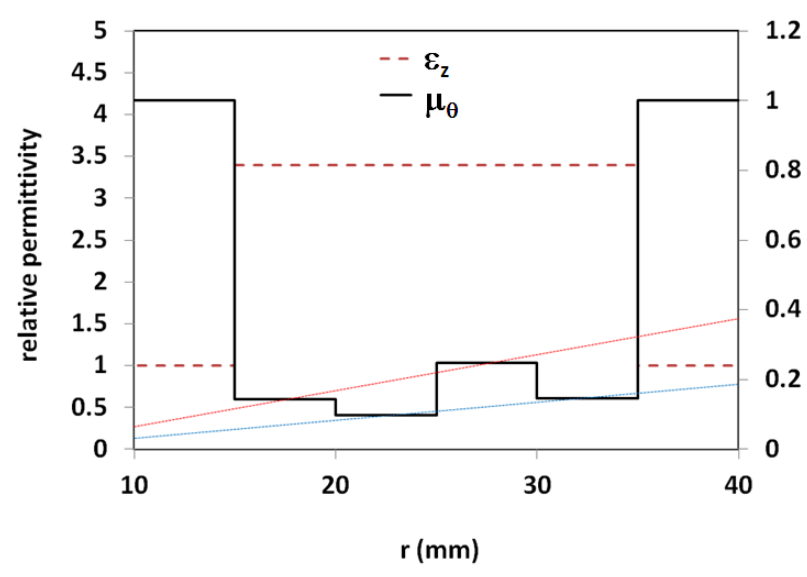

Fig. 7. Reduced model parameters for practical implementation as a function of the radial distance (center of the RPC located at $r=0 \mathrm{~mm}$ ). A four layer device is designed. Angular permeability (solid line) $\mu_{\alpha}(r)$ follows a stair-like profile bounded by layer $a$ and layer $b$ analytical parameter functions (dotted lines); permittivity (dashed line) $\varepsilon_{z}=3.4$ for the whole RPC shell; radial permeability $\mu_{r}$ (not plotted) is constant and equal to that of the background $\left(\mu_{r}=1\right)$.

reduced profile shell from the behavior of the ideal profile shell. A comparison and validation of this result is given in the following for a specific ideal RPC device that is compared to a physically implementable reduced profile RPC.

With these premises, a set of reduced constitutive parameter functions is illustrated in Fig. 7. This graph describes the parameter values for a four layer RPC, taking into account that the inner cavity and external background are air $(\mu=\varepsilon=1)$. Actually, only two of the parameters are effectively designed: $\mu_{d}(r)$ and $\varepsilon_{z}(r)$. In addition, permittivity is constant over the radial dimension for the four layers $\left(\varepsilon_{z}(r)=\varepsilon_{z_{z} \text { red }}\right)$. The goal of this configuration is to maintain a resonant response in a similar frequency range with respect to the RPC described in Fig. 2. The dispersion diagram for an equivalent ideal RPC is therefore different from the one provided in Fig. 1. The angular permeability $\mu_{d}(r)$ is implemented with a stair like profile starting from the values given in Fig. 2 and dividing them by a factor ten. The $\mu_{d}(r)$ decrease is performed because $\mu_{r}(r)$ takes a constant value of one (much higher than those of Fig. 2). This interplay tends to approximately preserve a similar resonant frequency range. Starting from the continuous dotted curves of Fig. 7, within each layer, $\mu_{d}(r)$ is constant and equal to the respective ( $a$ or $b$ ) layer value at its center:

$\mu_{\theta} \quad r=\left\{\begin{array}{l}\mu_{\theta a} \quad r=\mu_{\theta a}\left(r_{\text {int }}+\frac{d_{a}}{2}+n-1 d\right) \\ \mu_{\theta b} \quad r=\mu_{\theta b}\left(r_{\text {int }}+\frac{3 d_{b}}{2}+n-1 d\right)\end{array} n=1,2\right.$.

Each layer is $5 \mathrm{~mm}$ wide $\left(d_{a}=d_{b}=5 \mathrm{~mm}\right)$. The permittivity function has a constant value of $\varepsilon_{z}(r)=\varepsilon_{\varepsilon_{-} \text {red }}=3.4$, for all four layers, which is close to the mean value already used in Table II. Finally, the function for the radial permeability $\mu_{r}(r)$ is not implemented and this parameter remains equal to the background permeability $\left(\mu_{r_{-} \text {red }}=1\right)$. In summary, this
TABLE III

COMPARISON OF RESONANT FREQUENCIES FOR TWO FOUR LAYER REDUCED AND FULL PARAMETER SETS RPCS

\begin{tabular}{|c|c|c|c|c|}
\hline $\begin{array}{l}\text { Mode } \\
\text { pattern }\end{array}$ & $\begin{array}{c}\text { Mode } \\
\text { symmetry }\end{array}$ & $\begin{array}{c}\text { Full profile } \\
\text { RPC } f_{r}(\mathrm{GHz})\end{array}$ & $\begin{array}{l}\text { Reduced profile } \\
\text { RPC } f_{r}(\mathrm{GHz})\end{array}$ & $\begin{array}{r}\text { Relative } \\
\text { difference }\end{array}$ \\
\hline & $\begin{array}{l}\text { shell Bragg } \\
q=2 m=1\end{array}$ & 2.889 & 2.735 & $5.33 \%$ \\
\hline & $\begin{array}{l}\text { shell Bragg } \\
q=3 m=1\end{array}$ & 3.897 & 3.803 & $2.41 \%$ \\
\hline & $\begin{array}{l}\text { shell Bragg } \\
q=4 m=1\end{array}$ & 4.943 & 4.830 & $2.3 \%$ \\
\hline & $\begin{array}{l}\text { shell Bragg } \\
q=5 m=1\end{array}$ & 6.041 & 5.781 & $4.3 \%$ \\
\hline
\end{tabular}

Constitutive parameters for the full profile RPC are the following $\left(\mu_{\mathrm{ra}}{ }^{-1}\right.$, $\left.\mu_{\theta a}, \varepsilon_{z a}{ }^{-1}\right)=(0.347 r / d, 0.08 r / d, 0.143 r / d)$ and $\left(\mu_{r b}{ }^{-1}, \mu_{b b}, \varepsilon_{z b}{ }^{-1}\right)=(0.5 r / d$, ${ }_{1} 0.04 r / d, 0.1 r / d$ ). Constitutive parameters for the reduced profile RPC are given in Fig. 7. Resonant modes cover only the frequency range from 2.5 to $6.5 \mathrm{GHz}$.

configuration maintains the angular permeability distribution and uses a mean value for the permittivity across all layers of the ideal device. A comparison of this device (especially because of having $\mu_{r}=1$ ) with the initial ideal device (with the much lower Fig. 2 values) makes that resonant shell modes shift to lower frequency values.

As pointed out previously, a comparison can be performed between the reduced profile RPC described in Fig. 7 and an ideal RPC (both of same size). The comparison in terms of resonant frequencies is given in Table III, together with the constitutive parameters of the equivalent ideal shell. The constitutive parameters of this second ideal RPC are derived from Equations (12) and (13). This comparison confirms the approximate equivalence between both shells with differences in the resonant frequencies around or below 5\% for all the resonant modes in the frequency range from 2.7 to $6.5 \mathrm{GHz}$. In this reported frequency range, only first order radial Bragg resonances are obtained. They have different angular symmetry patterns, and this behavior is consistent in both shells.

\section{B. Practical design of a reduced profile RPC}

For a practical implementation of the profile we have selected a unitary cell composed of a resonant split ring resonator (SRR) particle, as proposed in [41] and different from [32]. This unitary component has reduced bianisotropy effects compared for example with the SRR proposed in [8]. This is important due to the fact that it is not desired that the array of resonators implementing the $\mu_{d}(r)$ function interferes with the $\mu_{r}(r)$ function (which in our case is neutral). General permeability of an array of SRRs follows a Lorentz-like model with the resonant frequency separating positive and negative values of effective permeability, [8, 42]. With the proper design of the geometric dimensions of the SRRs it is possible to define the permeability response at a specific frequency (the design frequency). An optimization process is required to 

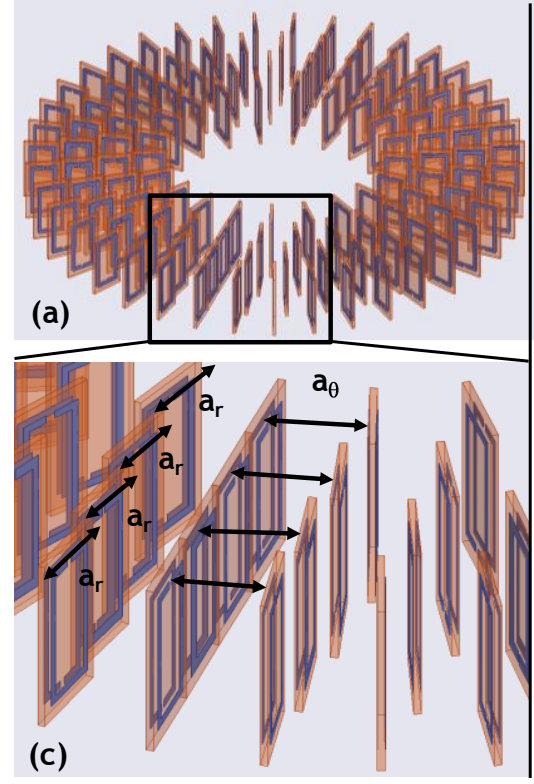

(b)
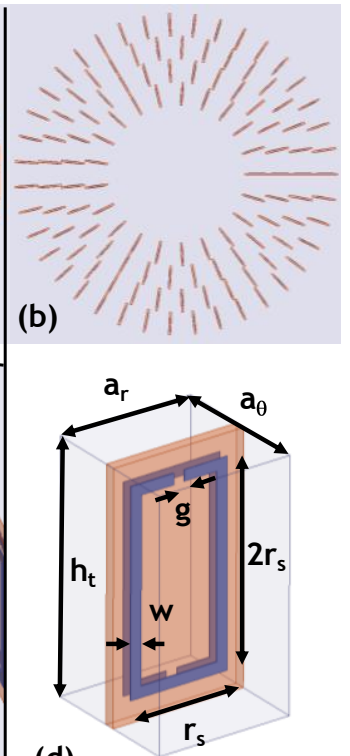

(d)

Fig. 8. Reduced model implementation of a fours layers simplified microstructure. Radially oriented SRRs implement the $\mu_{\theta}(r)$ profile as in Fig. 7. (a) four layers microstructure with internal cavity radius $r_{\text {int }}=15 \mathrm{~mm}$ and an integer number of Split Ring Resonators (SRRs) per layer; (b) top view; (c) radial and angular cell parameters in a zoomed view; (d) SRR unit cell configuration with design parameters.

match the desired permeability and permittivity values at the desired operation frequency. It is well known that for SRRs losses exist and are especially important for the frequencies close to the resonance frequency (due to the Lorentz-like model). This is especially critical when negative permeability values need to be implemented. Nevertheless, the proposed design (see Fig. 7) uses values of $\mu_{\phi}(r)$ that are positive and below 1. It is possible to take advantage of a permeability range (in the Lorentz model) where the frequency dispersion is not as high as near the resonance frequency of the SRRs. At the same time, this reduces the losses for the SRR response, with respect to other applications. Anyway, material losses derived from the use of copper and the dielectric material substrate are unavoidable. Copper has a finite conductivity $\sigma=$ $5.8 \times 10^{7} \mathrm{~S} / \mathrm{m}$.

Figure 8 depicts the scheme of the implemented design, together with the main dimensions. Figures 8(a) and 8(b) show oblique and top views of the reduced RPC structure implemented through four concentric layers of SRRs. Each layer is 'homogeneous' (i.e. all SRRs forming one layer are identical) and implements the specific set of constitutive parameter values of Table IV. Basically, the values of $\mu_{\phi}(r)$ and $\varepsilon_{z}=3.4$ are reproduced for each layer. Figure 8(c) displays a zoomed view of the SRR arrangement with equal radial thickness for each layer (radial periodicity $a_{r}$ ). This design is based on having an integer number of SRRs per layer and a constant angular separation between adjacent SRRs of the same layer (angular periodicity $a_{\theta}$ ). The relationship between both parameters is $a_{\theta}=\pi / 3 \times a_{r}$, guaranteeing that every concentric layer has six SRRs in addition to the previous one (cf. Table IV). Each circumference of the reported mean radii
(17.5 to $32.5 \mathrm{~mm}$ ) contains an integer number of equally spaced SRRs with angular periodicity $a_{\theta}$ Figure 8(d) shows the actual simulation scheme employed to extract the constitutive parameters of one unit cell. It is composed of a broadside coupled SRR, [43], formed by two metallic and symmetric split rings patterned on both sides of dielectric plate. Dielectric material is Neltec 9220 of low permittivity and low loss $\left(\varepsilon_{r}=2.2\right.$ and $\left.\tan \delta=0.0009\right)$. Note that periodic boundary conditions mimic the presence of a complete array of identical elements with the corresponding periodicity constant (in this case $a_{\theta}$ for the angular permeability). The constitutive parameters have been extracted using the standard Nicolson-Ross-Weir procedure, [44, 45], adapted as in [19]. These extracted parameters are also given in Table IV, together with the relative difference with respect to the target parameters of Fig. 7. These differences are in general lower than $1 \%$, a value comparable to the accuracy in the numerical simulations. The unit cell has been simulated taking into account both possible incidence directions (with respective periodicity parameters $a_{r}$ and $a_{\theta}$ ). Although it is a Cartesian unit cell, it is assumed that it reproduces accurately the extracted parameters in cylindrical coordinates. This agreement is obviously better for the largest radius layers. If the $\mathrm{H}$-field is polarized perpendicular to the SRR plane, we obtain $\mu_{\theta}$ and $\varepsilon_{z}$. If the $\mathrm{H}$-field is polarized parallel to the SRR plane, we obtain the unwanted radial permeability response of the SRR array and again a $\varepsilon_{z}$ permittivity value (which is quite close to the previous one). Fortunately, this unwanted magnetic response is very low (with $\mu_{r}$ values very close to 1 ), basically due to the fact that this SRR resonant particle has a low bianisotropic activity [41]. By this means, the expected $\mu_{r}$ target value is also obtained.

Using this scheme, only two parameters are needed to reproduce the targeted $\mu_{\theta}$ and $\varepsilon_{z}$ values: gap width $g$ of split in the rings and the metallic track width $w$ (see Fig. 8(d)). These parameters can be tuned with accuracy compatible with standard fabrication techniques, allowing the fabrication of a prototype.

\section{COMPARATIVE ANALYSIS OF THE REDUCED MODEL}

The analysis of the reduced prototype has been performed in two parallel ways, allowing for comparison of the obtained results and validation of the functionality of the designed structure.

In a first approach, the reduced shell has been numerically studied by defining analytically the anisotropic material forming each one of the four layers. It is an equation-based definition of the constitutive parameters. This is a 2D simulation where the parameter functions in Fig. 7 are directly assigned to the multilayer domains of the finite element solver, [34]. The RPC with constitutive parameters analytically defined effectively behaves as a resonator with shell Bragg resonances and also inner cavity resonances. Since the reduced parameter set is different than the one in Fig. 2, it is obvious that the resonant frequencies of the new resonator will not correspond to those of Table I, but rather to those of 
Table III. The study of the resonant modes of this microstructure was summarized there and all the microstructure modes between $2.7 \mathrm{GHz}$ and $6.5 \mathrm{GHz}$ are listed. We have therefore selected a $q=3$ symmetry (sextupolar) mode pattern that resonates at $f=3.8 \mathrm{GHz}$, and used it as our design frequency. It is evident that the mode richness of the reduced structure is much smaller than in the initial RPC (see Table I). Using a thickness of only four layers (instead of ten) limits the order and number of the shell Bragg resonances. Let us also mention that at this operation frequency, free space wavelength is $\lambda(3.8 \mathrm{GHz})=79 \mathrm{~mm}$, which is much larger than the layer thickness $d_{a}=d_{b}=5 \mathrm{~mm}$.

The second approach consisted of implementing the RPC structure at the selected resonant frequency $(f=3.8 \mathrm{GHz})$, but using a SRR configuration as depicted in Fig. 8. A layer by layer design is performed by optimizing each device unit cell as it was illustrated in Table IV. Then, the complete structure is numerically analyzed with a different $3 \mathrm{D}$ finite element solver, [46]. The approach is based on the simulation of discrete SRR resonators to implement the designed geometry instead of defining the RPC directly from analytical equations for the constitutive parameters. The ab-initio simulations have been performed only at the design frequency $3.8 \mathrm{GHz}$ because of the computational resources required, but the results should be close to what is expected in a measurement setup at this frequency. Let us recall that this structure is designed for $\mathrm{TM}^{\mathrm{z}}$ polarization. The 3D simulation domain, if it has to be compared to the 2D simulation results of the first approach, is bounded on the $z$-axis by Perfect Electric Conductor (PEC) boundaries. Therefore, this configuration mimics a $z$-invariant simulation domain. One important note about this second approach is the fact that the SRR behavior is inherently dispersive, with a Lorentz-like function for each array of elements in the multilayers. This makes it so that the values of both $\mu_{\theta}$ and $\varepsilon_{z}$ are valid only in a frequency range close to the design frequency. On the one hand, it is a bandwidth limited approach, but on the other hand, the selected values of $\mu_{\theta}$ and $\varepsilon_{z}$ are not close to the highly dispersive values obtained near the resonance frequency of the SRRs (i.e. $\mu_{\theta}$ and $\varepsilon_{z}$ have smooth variations around the design frequency).

Two configurations have been tested and compared in terms of the resonant response of a reduced model RPC when interacting with an external excitation. Each configuration is compared using both numerical approaches: analytical parameters and ab-initio simulations.

\section{A. Line source excitation}

The first configuration is designed to verify the interaction of a line source placed close to the RPC. The E-field magnitude patterns in the region around the RPC are displayed in Fig. 9. The RPC is centered on the origin of the radial coordinate system and the line source is located at position $x_{0}$ $=y_{0}=40 \mathrm{~mm}$. Figure 9(a) shows the result for an equation based parameters model simulation and Fig. 9(b) shows the result for the SRR implemented RPC (cut plane plot of the 3D simulation). The color scale represents E-field magnitude with same range in both plots. From this comparison, it is clear that
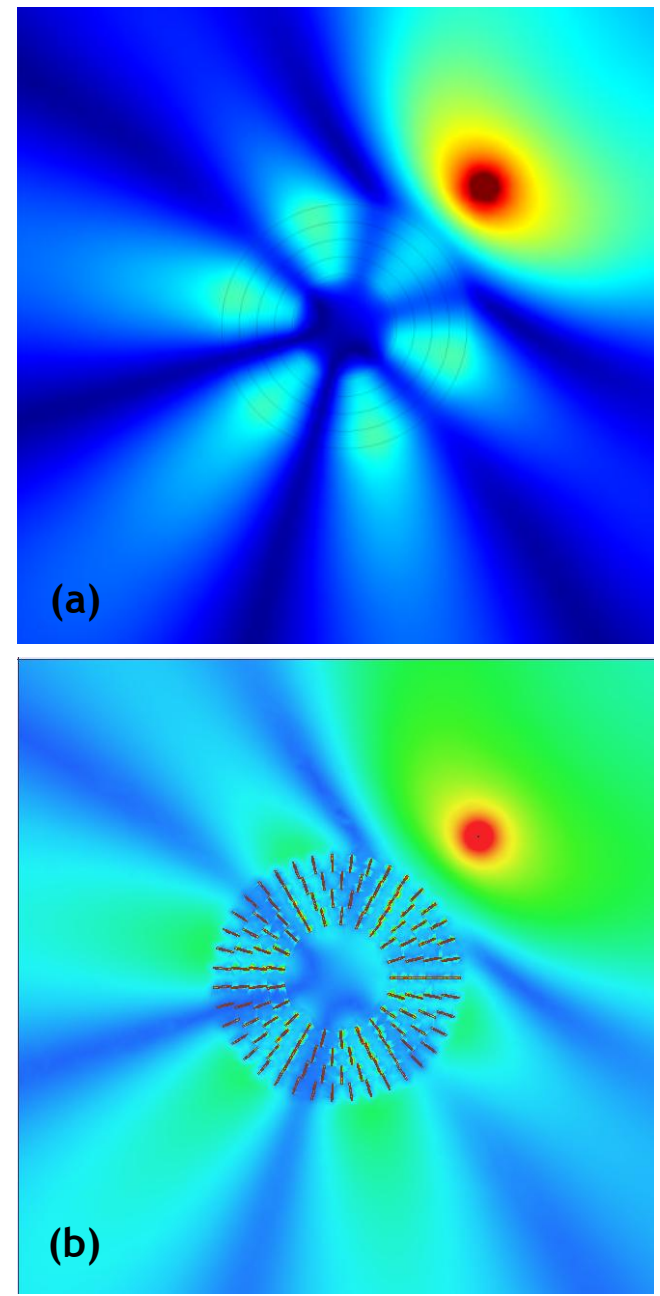

Fig. 9. E-field magnitude plots of an external line source exciting the $q=3$ resonance of a simplified RPC at $f=3.8 \mathrm{GHz}$. (a) full wave simulation from an analytical definition of the constitutive parameters; (b) full wave (abinitio) simulation of the reduced model implementation on an horizontal cut plane. Resonance pattern associated to the $q=3$ mode is apparent in both results inside and outside the RPC shell.

the $q=3$ mode is excited in the RPC shell at the predicted resonance frequency, $f=3.8 \mathrm{GHz}$. Field maxima and minima are closely comparable in both simulations and orientation of the mode pattern towards the source is preserved. Within the RPC shell, the dielectric plates between the metallic tracks of the SRRs concentrate large amounts of electric field, but overall the field patterns closely follow the 'envelope' corresponding to the $q=3$ field pattern.

\section{B. Plane wave excitation}

Finally, in a second configuration the reduced profile RPC shell is illuminated by a plane wave. The excitation wave has a normalized amplitude of $1 \mathrm{~V} / \mathrm{m}$ and the E-field is polarized in the $z$-direction and propagates in the $\mathrm{x}$-direction (see Fig. 10). Again, the two analysis methods are compared, and results in terms of E-field magnitude of the total field are displayed. Figure 10(a) corresponds to the equation-based solution and Fig. 10(b) corresponds to the ab-initio simulation. It is possible to observe the very close similitude between both panels with respect to the scattered field outside the shell, as 

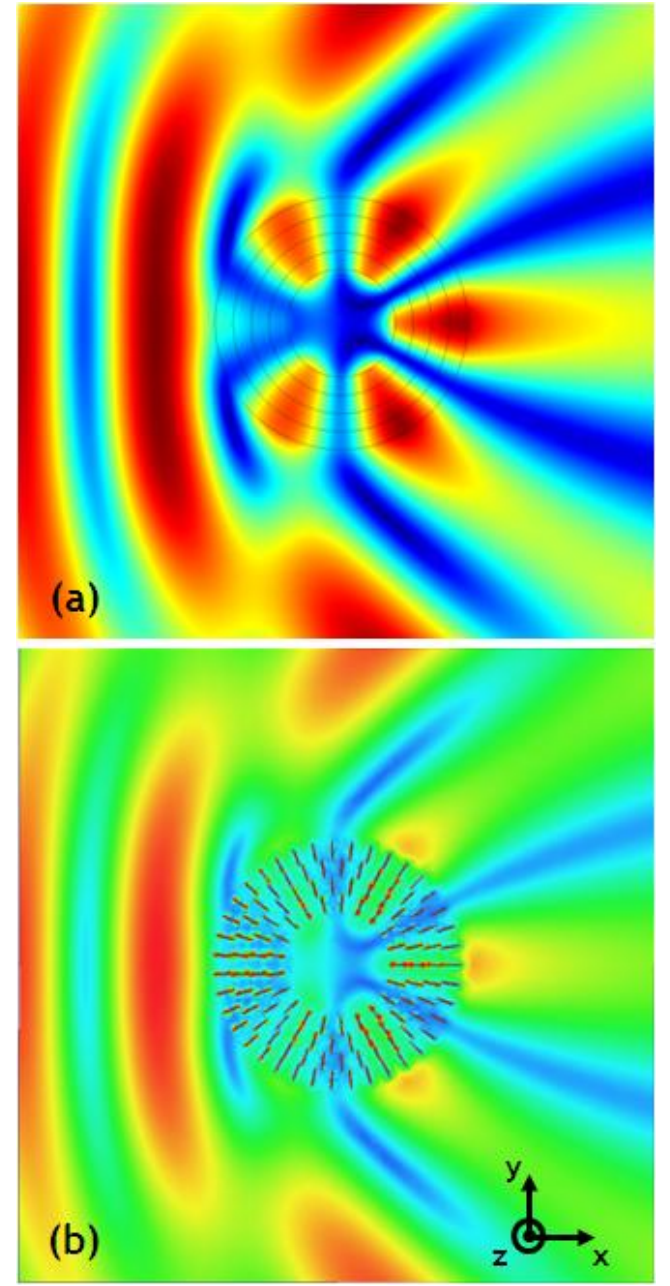

Fig. 10. E-field magnitude plots of a plane wave exciting the $q=3$ resonance of a simplified RPC at $f=3.8 \mathrm{GHz}$ (incidence in the x direction; normalized amplitude $E^{z}=1 \mathrm{~V} / \mathrm{m}$ ). (a) full wave simulation from an equation based definition of the constitutive parameters; (b) full wave (ab-initio) simulation of the reduced model implementation on an horizontal cut plane. Resonance pattern associated to the $q=3$ mode is apparent in both results inside and outside the RPC shell.

well as for the resonant mode within the shell itself. The $q=3$ resonant mode pattern at $f=3.8 \mathrm{GHz}$ is clearly apparent in both graphs, with clearly defined maxima and minima in both approaches. Finally, let us note that again the resonant mode pattern points towards the source of the incident wave.

\section{CONCLUSION}

A comprehensive analysis of Radial Photonic Crystal structures has been performed. RPCs can be basically described as periodic media that verify Bloch's theorem. They are implemented with radially dependent constitutive parameters making them systems with unique characteristics. These 'very ordered' structures have been analyzed starting from their ideal profile configuration and showing their possible application as resonant structures. Their combination with radiation sources allows the analysis of potential interactions. We have pointed out possible applications of RPCs as beam-forming shells for line sources. Also, their application as frequency and position sensors is outlined and explicitly demonstrated with a simulation example. Additionally, in view of a practical implementation, a reduced profile has been investigated. It is showed that it is possible to retain part of the RPC functionality even if the complexity of the device is reduced and hence is affordable in terms of standard fabrication techniques. Some ab-initio simulations demonstrate that the RPC can be implemented in practice by using split ring resonator arrangements. In particular, a multilayered microstructure synthesizing a radially dependent angular permeability has been designed and compared to the equation-based parameters model. Results have been obtained in this case for the interaction with a line source and a plane wave. This study opens the path to further works on both the analysis of specific RPC designs and the fabrication and measurement of a RPC prototype.

\section{APPENDIX}

\section{ANISOTROPIC EQUATION IN CYLINDRICAL COORDINATES}

Starting from the Maxwell equations (without sources and taking into account anisotropic constitutive parameters), the divergence equations for displacement and inductance fields are:

$$
\begin{aligned}
& \nabla \cdot \vec{D}=0 \rightarrow \nabla \cdot[\overrightarrow{\underline{\varepsilon}} \cdot \vec{E}]=0 \\
& \nabla \cdot \vec{B}=0 \rightarrow \nabla \cdot[\overline{\bar{\mu}} \cdot \vec{H}]=0
\end{aligned}
$$

and the field coupling equations:

$$
\begin{aligned}
& \nabla \times \vec{E}+i \omega \bar{\mu} \cdot \vec{H}=0 \\
& \nabla \times \vec{H}-i \omega \bar{\varepsilon} \cdot \vec{E}=0
\end{aligned}
$$

We may assume here that our problem is strictly a $2 \mathrm{D}$ one and partial derivatives with respect to $z$ are null $(\delta / \delta z=0)$. Also, definition of anisotropic constitutive parameters corresponds to diagonal tensors (in the cylindrical coordinate system), that only have a radial dependence (cf. Section II):

$$
\begin{aligned}
& \bar{\mu}=\left(\begin{array}{ccc}
\mu_{r} r & 0 & 0 \\
0 & \mu_{\theta} r & 0 \\
0 & 0 & \mu_{z}
\end{array}\right)
\end{aligned}
$$

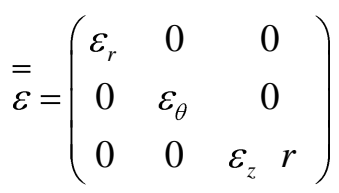

If we limit ourselves to $\mathrm{TM}^{\mathrm{z}}$ modes $\left(\mathrm{E}_{\mathrm{z}}\right.$ modes), we are looking for solutions with the following functional form:

$$
\begin{aligned}
& \vec{H}=H_{r} r, \theta, H_{\theta} r, \theta, 0 \\
& \vec{E}=0,0, E_{z} \quad r, \theta
\end{aligned}
$$

where in the case of the E-field the $(r, \theta)$ dependence is factorized as:

$$
\vec{E} r, \theta=\sum_{q} E_{q} r e^{i q \theta} \widehat{z}
$$


Developing equations [17] and [18], and separating each one of the three cylindrical coordinates $(r, \theta, z)$ :

$$
\begin{aligned}
& \frac{1}{r} \frac{\partial E_{z}}{\partial \theta}+i \omega \mu_{r} H_{r}=0 \\
& -\frac{\partial E_{z}}{\partial r}+i \omega \mu_{\theta} H_{\theta}=0 \\
& \frac{1}{r}\left[\frac{\partial}{\partial \theta} r E_{\theta}-\frac{\partial E_{r}}{\partial \theta}\right]+i \omega \mu_{z} H_{z}=0 \\
& \frac{1}{r} \frac{\partial H_{z}}{\partial \theta}-i \omega \varepsilon_{r} E_{r}=0 \\
& -\frac{\partial H_{z}}{\partial r}-i \omega \varepsilon_{\theta} E_{\theta}=0 \\
& \frac{1}{r}\left[\frac{\partial}{\partial r} r H_{\theta}-\frac{\partial H_{r}}{\partial \theta}\right]-i \omega \varepsilon_{z} E_{z}=0
\end{aligned}
$$

From Eq. (23), we get:

$$
H_{r} r, \theta=i \frac{1}{\omega \mu_{r} r} \frac{\partial E_{z}}{\partial \theta}
$$

and from Eq. (24), we get:

$$
H_{\theta} r, \theta=-i \frac{1}{\omega \mu_{\theta}} \frac{\partial E_{z}}{\partial r}
$$

Replacing these two $\mathrm{H}$-field components in Eq, (28) to obtain an E-field equation ( $\mathrm{TM}^{\mathrm{z}}$ or $\mathrm{E}_{\mathrm{z}}$ modes) gives:

$$
\frac{1}{r}\left[\frac{\partial}{\partial r}\left(-i \frac{r}{\omega \mu_{\theta}} \frac{\partial E_{z}}{\partial r}\right)-\frac{\partial}{\partial \theta}\left(i \frac{1}{\omega \mu_{r} r} \frac{\partial E_{z}}{\partial \theta}\right)\right]-i \omega \varepsilon_{z} E_{z}=0
$$

Re-arranging this equation gives:

$$
\frac{1}{r} \frac{\partial}{\partial r}\left(\frac{r}{\mu_{\theta} r} \frac{\partial E_{z}}{\partial r}\right)+\frac{1}{\mu_{r} r r^{2}} \frac{\partial^{2} E_{z}}{\partial \theta^{2}}+\omega^{2} \varepsilon_{z} r E_{z}=0
$$

Now, replacing the $E_{z}(r, \theta)$ field expression given by Eq. (22), we arrive to the anisotropic Eq. (2) for $E_{q}(r)$. Finally, the general solution of Eq. (2) is:

$$
E_{q} r=\sum_{G} e^{i k r} e^{i G r},
$$

where the reciprocal lattice vectors are $G=2 \pi q / d$, and $k$ represents the Bloch wave vector.

\section{ACKNOWLEDGMENT}

The authors gratefully acknowledge the assistance of Kimberly Moore in the revision of this manuscript.

\section{REFERENCES}

[1] G. V. Eleftheriades and K. G. Balmain, Negative-Refraction Metamaterials: Fundamental Principles and Applications. Piscataway, NJ: Wiley - IEEE Press, 2005.

[2] N. Engheta and R. W. Ziolkowski, Metamaterials: Physics and Engineering Explorations. Piscataway, NJ: Wiley - IEEE Press, 2006.
[3] R. Marqués, F. Martín and M. Sorolla, Metamaterials with Negative Parameter: Theory, Design, and Microwave Applications. Piscataway, NJ: Wiley - Interscience, 2008.

[4] D. Schurig, J. J. Mock, B. J. Justice, S. A. Cummer, J. B. Pendry, A. F. Starr and D. R. Smith, "Metamaterial electromagnetic cloak at microwave frequencies," Science, vol. 314, pp. 977-980, NOV 10, 2006.

[5] D. Smith, W. Padilla, D. Vier, S. Nemat-Nasser and S. Schultz, "Composite medium with simultaneously negative permeability and permittivity," Phys. Rev. Lett., vol. 84, pp. 4184-4187, MAY 1, 2000.

[6] E. E. Narimanov and A. V. Kildishev, "Optical black hole: Broadband omnidirectional light absorber," Appl. Phys. Lett., vol. 95, pp. 041106, JUL 27, 2009.

[7] J. B. Pendry, "Negative refraction makes a perfect lens," Phys. Rev. Lett., vol. 85, pp. 3966-3969, OCT 30, 2000.

[8] J. B. Pendry, A. J. Holden, D. J. Robbins and W. J. Stewart, "Magnetism from conductors and enhanced nonlinear phenomena," IEEE Trans. Microwave Theory Tech., vol. 47, pp. 2075-2084, NOV, 1999.

[9] M. Saadoun and N. Engheta, "A Reciprocal Phase-Shifter using Novel Pseudochiral Or Omega-Medium," Microwave Opt Technol Lett, vol. 5, pp. 184-188, APR, 1992.

[10] H. Chen, L. Ran, J. Huangfu, X. Zhang, K. Chen, T. Grzegorczyk and J. Kong, "Negative refraction of a combined double S-shaped metamaterial," Appl. Phys. Lett., vol. 86, pp. 151909, APR 11, 2005.

[11] J. D. Baena, L. Jelinek and R. Marques, "Towards a systematic design of isotropic bulk magnetic metamaterials using the cubic point groups of symmetry," Physical Review B, vol. 76, pp. 245115, DEC, 2007.

[12] R. Marques, J. Martel, F. Mesa and F. Medina, "A new 2D isotropic lefthanded metamaterial design: Theory and experiment," Microwave Opt Technol Lett, vol. 35, pp. 405-408, DEC 5, 2002.

[13] R. Shelby, D. Smith, S. Nemat-Nasser and S. Schultz, "Microwave transmission through a two-dimensional, isotropic, left-handed metamaterial," Appl. Phys. Lett., vol. 78, pp. 489-491, JAN 22, 2001.

[14] I. Gil, J. Garcia-Garcia, J. Bonache, F. Martin, M. Sorolla and R. Marques, "Varactor-loaded split ring resonators for tunable notch filters at microwave frequencies," Electron. Lett., vol. 40, pp. 1347-1348, OCT 14, 2004.

[15] J. Carbonell, V. E. Boria and D. Lippens, "Nonlinear effects in split ring resonators loaded with heterostructure barrier varactors," Microwave Opt Technol Lett, vol. 50, pp. 474-479, FEB, 2008.

[16] A. D. Boardman, V. V. Grimalsky, Y. S. Kivshar, S. V. Koshevaya, M. Lapine, N. M. Litchinitser, V. N. Malnev, M. Noginov, Y. G. Rapoport and V. M. Shalaev, "Active and tunable metamaterials," Laser Photon. Rev., vol. 5, pp. 287-307, MAR, 2011.

[17] Q. Cheng, T. J. Cui, W. X. Jiang and B. G. Cai, "An omnidirectional electromagnetic absorber made of metamaterials," New Journal of Physics, vol. 12, pp. 063006, JUN 3, 2010.

[18] F. Zhang, S. Potet, J. Carbonell, E. Lheurette, O. Vanbesien, X. Zhao and D. Lippens, "Negative-Zero-Positive Refractive Index in a Prism-Like Omega-Type Metamaterial," IEEE Trans. Microwave Theory Tech., vol. 56, pp. 2566-2573, NOV, 2008.

[19] D. R. Smith, D. C. Vier, T. Koschny and C. M. Soukoulis, "Electromagnetic parameter retrieval from inhomogeneous metamaterials," Physical Review E, vol. 71, pp. 036617, MAR, 2005.

[20] X. Chen, T. Grzegorczyk, B. Wu, J. Pacheco and J. Kong, "Robust method to retrieve the constitutive effective parameters of metamaterials," Physical Review E, vol. 70, pp. 016608, JUL, 2004.

[21] Z. Szabo, G. Park, R. Hedge and E. Li, "A Unique Extraction of Metamaterial Parameters Based on Kramers-Kronig Relationship," IEEE Trans. Microwave Theory Tech., vol. 58, pp. 2646-2653, OCT, 2010.

[22] J. Baena, J. Bonache, F. Martin, R. Sillero, F. Falcone, T. Lopetegi, M. Laso, J. Garcia-Garcia, I. Gil, M. Portillo and M. Sorolla, "Equivalentcircuit models for split-ring resonators and complementary split-ring resonators coupled to planar transmission lines," IEEE Trans. Microwave Theory Tech., vol. 53, pp. 1451-1461, APR, 2005.

[23] L. J. Rogla, J. Carbonell and V. E. Boria, "Study of equivalent circuits for open-ring and split-ring resonators in coplanar waveguide technology," IET Microwaves Antennas \& Propagation, vol. 1, pp. 170-176, FEB, 2007.

[24] N. Horiuchi, Y. Segawa, T. Nozokido, K. Mizuno and H. Miyazaki, "Isotropic photonic gaps in a circular photonic crystal," Opt. Lett., vol. 29, pp. 1084-1086, MAY 15, 2004.

[25] N. Horiuchi, Y. Segawa, T. Nozokido, K. Mizuno and H. Miyazaki, "High-transmission waveguide with a small radius of curvature at a bend fabricated by use of a circular photonic crystal," Opt. Lett., vol. 30, pp. 973-975, MAY 1, 2005. 
[26] S. Xiao and M. Qiu, "High-Q microcavities realized in a circular photonic crystal slab," Photonics and Nanostructures-Fundamentals and Applications, vol. 3, pp. 134-138, DEC, 2005.

[27] D. Torrent and J. Sanchez-Dehesa, "Radial Wave Crystals: Radially Periodic Structures from Anisotropic Metamaterials for Engineering Acoustic or Electromagnetic Waves," Phys. Rev. Lett., vol. 103, pp. 064301, AUG 7, 2009.

[28] D. Torrent and J. Sanchez-Dehesa, "Acoustic resonances in twodimensional radial sonic crystal shells," New Journal of Physics, vol. 12, pp. 073034, JUL 27, 2010.

[29] J. Carbonell, D. Torrent, A. Diaz-Rubio and J. Sanchez-Dehesa, "Multidisciplinary approach to cylindrical anisotropic metamaterials," New J. Phys., vol. 13, pp. 103034, OCT 24, 2011.

[30] R. F. Harrington, Time-Harmonic Electromagnetic Fields. Chap. 5, pp. 198-199, New York, NY: IEEE Press - Wiley Interscience, 1961.

[31] B. Ivsic, Z. Sipus and S. Hrabar. Analysis of uniaxial multilayer cylinders used for invisible cloak realization. IEEE Transactions on Antennas and Propagation 57(5), pp. 1521-1527. 2009.

[32] J. G. Pollock and A. K. Iyer. Effective-medium properties of cylindrical transmission-line metamaterials. IEEE Antennas and Wireless Propagation Letters 10pp. 1491-1494. 2011.

[33] L. M. Brekhovskikh and R. T. Beyer, Waves in Layered Media. New York, NY: Academic Press New York, 1980.

[34] Comsol AB (Sweden), "Comsol Multiphysics (v. 4.1)," 2010.

[35] T. Ochiai and J. Sanchez-Dehesa, "Localized defect modes in finite metallic two-dimensional photonic crystals," Physical Review B, vol. 65, pp. 245111, JUN 15, 2002.

[36] A. Kitagawa and J. Sakai. Bloch theorem in cylindrical coordinates and its application to a Bragg fiber. Physical Review A, vol. 80(3), pp. 033802. 2009.

[37] J. Sakai. Optical power confinement factor in a bragg fiber: 1. formulation and general properties. Journal of the Optical Society of America B-Optical Physics, vol. 24(1), pp. 9-19. 2007.

[38] S. Arslanagic, R. W. Ziolkowski and O. Breinbjerg, "Analytical and numerical investigation of the radiation from concentric metamaterial spheres excited by an electric Hertzian dipole," Radio Sci., vol. 42, pp. RS6S16, OCT 19, 2007.

[39] S. Arslanagic, R. W. Ziolkowski and O. Breinbjerg, "Excitation of an electrically small metamaterial-coated cylinder by an arbitrarily located line source," Microwave Opt Technol Lett, vol. 48, pp. 2598-2606, DEC, 2006.

[40] G. B. Arfken. Mathematical Methods for Physicists (Chapter 9), San Diego, CA: Academic Press, 2001.

[41] R. Marques, F. Medina and R. Rafii-El-Idrissi, "Role of bianisotropy in negative permeability and left-handed metamaterials," Physical Review B, vol. 65 , pp. 144440, APR 1, 2002.

[42] J. Carbonell, L. J. Rogla, V. E. Boria and D. Lippens, "Design and experimental verification of backward-wave propagation in periodic waveguide structures," IEEE Trans. Microwave Theory Tech., vol. 54, pp. 1527-1533, APR, 2006.

[43] R. Marques, F. Mesa, J. Martel and F. Medina, "Comparative analysis of edge- and broadside-coupled split ring resonators for metamaterial design - Theory and experiments," IEEE Transactions on Antennas and Propagation, vol. 51, pp. 2572-2581, OCT, 2003.

[44] A. Nicolson and G. Ross, "Measurement of Intrinsic Properties of Materials by Time-Domain Techniques," IEEE Transactions on Instrumentation and Measurement, vol. IM19, pp. 377-382, 1970.

[45] W. Weir, "Automatic Measurement of Complex Dielectric-Constant and Permeability at Microwave-Frequencies," Proc IEEE, vol. 62, pp. 33-36, 1974.

[46] Ansoft, "High Frequency Structure Simulator (HFSS), v.14," 2012.

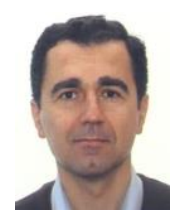

Jorge Carbonell was born in Valencia (Spain) in 1971. He received the MSc. in Telecommunications Engineering from the Universidad Politécnica de Valencia (Spain) in 1995, and the $\mathrm{PhD}$ degree in Electrical Engineering, European Label with Honors, from the University of Lille (France) in 1998.

From 1996 to 1998, he was with the Institut d'Electronique et de Microélectronique du Nord (IEMN) at the University of Lille (France), where his research activity included EM analysis of active and passive devices for space applications, and in particular photonic bandgap materials. From 1999 to 2003, he worked within the wireless industry with Ericsson, Siemens, Retevisión Móvil, and Telefónica Móviles. During that period he was involved in the design and deployment of second- and third-generation wireless communication systems and networks, and mainly focused on radio engineering. Since January 2004, he holds a research position at the Universidad Politécnica de Valencia (Spain). His current research activity concerns the analysis and design of passive periodic structures and metamaterials.

\section{Daniel Torrent}

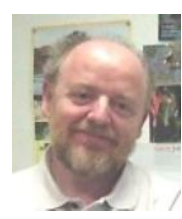

José Sánchez-Dehesa received the Ph.D. degree in Physics from the Universidad Autonoma de Madrid, Spain, in 1982. In 1983 he joined the Department of Physics at the University of Notre Dame, USA, as a research scientist. From 1985 till 2003 he was Associate Professor at the Condensed Matter Department in the Universidad Autonoma de Madrid. Since October 2003 he is Professor at the Electronics Engineering Department in the Universitat Politècnica de València, Spain. He has authored or coauthored around 200 research papers in peer-reviewed journals covering different topics in solid state physics, optics and acoustics. His current interest includes photonic and acoustic crystals, metamaterials and acoustic barriers. 